Supplementary Table S5

\begin{tabular}{|c|c|c|c|c|c|}
\hline ORF & Rank3 & $\begin{array}{c}\text { Log2Fold } \\
\text { Change }\end{array}$ & base Mean & IfcSE & padj \\
\hline k141_548983 & 2-oxoglutarate/malate translocator & $-1,53$ & 1387,78 & 0,15 & $2,6174 \mathrm{E}-21$ \\
\hline k141_333091 & 2-oxoglutarate/malate translocator & $-1,69$ & 101,41 & 0,18 & $3,84763 E-20$ \\
\hline k141_475239 & 2-oxoglutarate/malate translocator & $-1,45$ & 136,58 & 0,19 & $1,52129 \mathrm{E}-13$ \\
\hline k141_102628 & 2-oxoglutarate/malate translocator & 2,13 & 90,94 & 0,28 & $9,88886 \mathrm{E}-13$ \\
\hline k141_117083 & 2-oxoglutarate/malate translocator & $-0,93$ & 18863,85 & 0,15 & $1,31208 \mathrm{E}-08$ \\
\hline k141_452363 & 2-oxoglutarate/malate translocator & $-1,48$ & 32,04 & 0,25 & $5,06933 \mathrm{E}-08$ \\
\hline k141_179996 & 2-oxoglutarate/malate translocator & 2,33 & 71,88 & 0,41 & $1,57078 \mathrm{E}-07$ \\
\hline k141_371041 & 2-oxoglutarate/malate translocator & $-0,86$ & 4174,34 & 0,15 & $2,25676 \mathrm{E}-07$ \\
\hline k141_327423 & 2-oxoglutarate/malate translocator & 2,22 & 18,80 & 0,46 & $8,48587 \mathrm{E}-06$ \\
\hline k141_200378 & 2-oxoglutarate/malate translocator & $-1,60$ & 21,40 & 0,35 & $3,12594 \mathrm{E}-05$ \\
\hline k141_262791 & 2-oxoglutarate/malate translocator & 1,41 & 356,90 & 0,31 & $3,23282 \mathrm{E}-05$ \\
\hline k141_241719 & 2-oxoglutarate/malate translocator & 1,79 & 14,58 & 0,43 & 0,000128015 \\
\hline k141_260463 & 2-oxoglutarate/malate translocator & 3,47 & 2,84 & 0,87 & 0,000273131 \\
\hline k141_266022 & 2-oxoglutarate/malate translocator & $-0,88$ & 41,89 & 0,22 & 0,00028495 \\
\hline k141_408987 & 2-oxoglutarate/malate translocator & 2,24 & 4,02 & 0,59 & 0,000532837 \\
\hline k141_102291 & 2-oxoglutarate/malate translocator & $-2,68$ & 4,39 & 0,72 & 0,000768286 \\
\hline k141_319289 & $\begin{array}{l}\text { Branched-chain amino acid ABC transporter, } \\
\text { amino acid-binding protein (TC 3.A.1.4.1) }\end{array}$ & $-1,69$ & 31,18 & 0,22 & $6,90317 \mathrm{E}-13$ \\
\hline k141_156650 & Carbonic anhydrase (EC 4.2.1.1) & 2,74 & 53,82 & 0,24 & $3,91823 E-29$ \\
\hline k141_354975 & Carbonic anhydrase (EC 4.2.1.1) & 2,62 & 170,96 & 0,26 & $1,49879 \mathrm{E}-22$ \\
\hline k141_214223 & Carbonic anhydrase (EC 4.2.1.1) & 6,84 & 10,28 & 0,73 & $2,12509 \mathrm{E}-19$ \\
\hline k141_211486 & Carbonic anhydrase (EC 4.2.1.1) & $-6,55$ & 16,54 & 0,76 & $2,47027 \mathrm{E}-16$ \\
\hline k141_143280 & Carbonic anhydrase (EC 4.2.1.1) & 1,79 & 174,39 & 0,22 & $1,62045 \mathrm{E}-14$ \\
\hline k141_495726 & Carbonic anhydrase (EC 4.2.1.1) & 5,14 & 36,48 & 0,64 & $2,63411 \mathrm{E}-14$ \\
\hline k141_356359 & Carbonic anhydrase (EC 4.2.1.1) & $-6,00$ & 11,44 & 0,75 & $3,68066 \mathrm{E}-14$ \\
\hline k141_303879 & Carbonic anhydrase (EC 4.2.1.1) & $-4,03$ & 16,80 & 0,56 & $7,20381 \mathrm{E}-12$ \\
\hline k141_414077 & Carbonic anhydrase (EC 4.2.1.1) & 5,29 & 5,02 & 0,78 & $1,28543 E-10$ \\
\hline k141_459441 & Carbonic anhydrase (EC 4.2.1.1) & 1,46 & 42,79 & 0,24 & $1,26296 \mathrm{E}-08$ \\
\hline k141_216585 & Carbonic anhydrase (EC 4.2.1.1) & 4,66 & 16,41 & 0,82 & $1,19559 \mathrm{E}-07$ \\
\hline k141_363238 & Carbonic anhydrase (EC 4.2.1.1) & 4,95 & 2,77 & 0,87 & $1,20493 E-07$ \\
\hline k141_531878 & Carbonic anhydrase (EC 4.2.1.1) & 1,70 & 36,90 & 0,30 & $1,2272 \mathrm{E}-07$ \\
\hline k141_542525 & Carbonic anhydrase (EC 4.2.1.1) & 4,26 & 4,67 & 0,79 & $4,75144 \mathrm{E}-07$ \\
\hline k141_149506 & Carbonic anhydrase (EC 4.2.1.1) & 5,28 & 3,47 & 0,98 & $5,25191 \mathrm{E}-07$ \\
\hline k141_303356 & Carbonic anhydrase (EC 4.2.1.1) & $-1,93$ & 14,24 & 0,37 & $1,09638 \mathrm{E}-06$ \\
\hline k141_286844 & Carbonic anhydrase (EC 4.2.1.1) & 4,55 & 4,22 & 0,89 & 1,93903E-06 \\
\hline k141_434110 & Carbonic anhydrase (EC 4.2.1.1) & $-3,47$ & 47,22 & 0,68 & $2,35356 \mathrm{E}-06$ \\
\hline k141_184675 & Carbonic anhydrase (EC 4.2.1.1) & 3,89 & 15,47 & 0,78 & $3,86526 \mathrm{E}-06$ \\
\hline k141_444418 & Carbonic anhydrase (EC 4.2.1.1) & 3,35 & 16,91 & 0,68 & $6,09955 E-06$ \\
\hline k141_157322 & Carbonic anhydrase (EC 4.2.1.1) & 3,66 & 5,77 & 0,76 & $9,13241 \mathrm{E}-06$ \\
\hline k141_328623 & Carbonic anhydrase (EC 4.2.1.1) & 1,40 & 187,89 & 0,29 & $1,12499 \mathrm{E}-05$ \\
\hline k141_367283 & Carbonic anhydrase (EC 4.2.1.1) & 4,08 & 7,31 & 0,86 & $1,20994 \mathrm{E}-05$ \\
\hline k141_459203 & Carbonic anhydrase (EC 4.2.1.1) & 4,05 & 5,53 & 0,86 & $1,26198 \mathrm{E}-05$ \\
\hline k141_314915 & Carbonic anhydrase (EC 4.2.1.1) & 2,16 & 9,28 & 0,46 & 1,573E-05 \\
\hline k141_289263 & Carbonic anhydrase (EC 4.2.1.1) & $-2,85$ & 4,38 & 0,61 & 1,90999E-05 \\
\hline k141_96334 & Carbonic anhydrase (EC 4.2.1.1) & 4,77 & 2,45 & 1,03 & $2,09178 \mathrm{E}-05$ \\
\hline k141_68948 & Carbonic anhydrase (EC 4.2.1.1) & 1,82 & 687,89 & 0,40 & $2,70967 \mathrm{E}-05$ \\
\hline k141_484827 & Carbonic anhydrase (EC 4.2.1.1) & 4,55 & 3,01 & 1,00 & $2,96163 E-05$ \\
\hline k141_464700 & Carbonic anhydrase (EC 4.2.1.1) & 3,16 & 7,23 & 0,70 & $3,10582 \mathrm{E}-05$ \\
\hline k141_9518 & Carbonic anhydrase (EC 4.2.1.1) & 4,72 & 2,35 & 1,07 & 4,99506E-05 \\
\hline k141_318302 & Carbonic anhydrase (EC 4.2.1.1) & $-3,55$ & 5,64 & 0,81 & 5,17727E-05 \\
\hline
\end{tabular}


Supplementary Table S5

\begin{tabular}{|c|c|c|c|c|c|}
\hline ORF & Rank3 & Change & base Mean & IfcSE & padj \\
\hline k141_3059 & Carbonic anhydrase (EC 4.2.1.1) & 4,62 & 2,21 & 1,07 & $7,43748 \mathrm{E}-05$ \\
\hline k141_150193 & Carbonic anhydrase (EC 4.2.1.1) & 4,65 & 2,25 & 1,09 & $8,83906 \mathrm{E}-05$ \\
\hline k141_369195 & Carbonic anhydrase (EC 4.2.1.1) & 3,92 & 9,00 & 0,95 & 0,000169426 \\
\hline k141_566540 & Carbonic anhydrase (EC 4.2.1.1) & 3,45 & 5,94 & 0,84 & 0,000171725 \\
\hline k141_94851 & Carbonic anhydrase (EC 4.2.1.1) & 1,57 & 16,71 & 0,39 & 0,000203793 \\
\hline k141_213823 & Carbonic anhydrase (EC 4.2.1.1) & 3,82 & 4,48 & 0,96 & 0,000282481 \\
\hline k141_500524 & Carbonic anhydrase (EC 4.2.1.1) & 4,79 & 3,58 & 1,21 & 0,000318637 \\
\hline k141_178359 & Carbonic anhydrase (EC 4.2.1.1) & 4,80 & 2,47 & 1,22 & 0,000338775 \\
\hline k141_40985 & Carbonic anhydrase (EC 4.2.1.1) & $-4,62$ & 2,22 & 1,19 & 0,000389359 \\
\hline k141_45252 & Carbonic anhydrase (EC 4.2.1.1) & 2,44 & 6,52 & 0,63 & 0,000418807 \\
\hline k141_198190 & Carbonic anhydrase (EC 4.2.1.1) & $-2,46$ & 8,36 & 0,64 & 0,00050449 \\
\hline k141_328628 & Carbonic anhydrase (EC 4.2.1.1) & 4,73 & 2,35 & 1,24 & 0,000549087 \\
\hline k141_290108 & Carbonic anhydrase (EC 4.2.1.1) & 3,50 & 5,81 & 0,92 & 0,000558005 \\
\hline k141_21358 & Carbonic anhydrase (EC 4.2.1.1) & 3,52 & 4,85 & 0,94 & 0,000681963 \\
\hline k141_117901 & Carbonic anhydrase (EC 4.2.1.1) & 3,67 & 2,35 & 1,00 & 0,000828477 \\
\hline k141_547573 & Carbonic anhydrase (EC 4.2.1.1) & 4,94 & 2,72 & 1,34 & 0,000862505 \\
\hline k141_147667 & Carbonic anhydrase (EC 4.2.1.1) & 4,40 & 1,88 & 1,23 & 0,001199178 \\
\hline k141_273287 & Carbonic anhydrase (EC 4.2.1.1) & $-4,38$ & 1,88 & 1,25 & 0,001479676 \\
\hline k141_126164 & Carbonic anhydrase (EC 4.2.1.1) & 4,27 & 1,72 & 1,23 & 0,001669531 \\
\hline k141_219560 & Carbonic anhydrase (EC 4.2.1.1) & $-5,04$ & 2,97 & 1,49 & 0,002319709 \\
\hline k141_297216 & Carbonic anhydrase (EC 4.2.1.1) & 4,12 & 2,22 & 1,24 & 0,002657057 \\
\hline k141_392312 & Carbonic anhydrase (EC 4.2.1.1) & 3,51 & 1,50 & 1,11 & 0,004632651 \\
\hline k141_297940 & Carbonic anhydrase (EC 4.2.1.1) & 3,88 & 1,31 & 1,24 & 0,004884004 \\
\hline k141_365333 & Carbonic anhydrase (EC 4.2.1.1) & $-4,22$ & 1,67 & 1,37 & 0,005709171 \\
\hline k141_568317 & Carbonic anhydrase (EC 4.2.1.1) & 4,14 & 1,57 & 1,37 & 0,006635881 \\
\hline k141_176042 & Carbonic anhydrase (EC 4.2.1.1) & $-1,75$ & 5,42 & 0,58 & 0,007161562 \\
\hline k141_273684 & Carbonic anhydrase (EC 4.2.1.1) & 4,04 & 1,47 & 1,40 & 0,009697377 \\
\hline k141_212502 & Gamma-glutamyl hydrolase (EC 3.4.19.9) & 6,10 & 6,11 & 0,77 & $6,78516 \mathrm{E}-14$ \\
\hline k141_353437 & Gamma-glutamyl hydrolase (EC 3.4.19.9) & $-1,90$ & 45,80 & 0,34 & 2,20347E-07 \\
\hline k141_127527 & Gamma-glutamyl hydrolase (EC 3.4.19.9) & $-1,45$ & 18,35 & 0,39 & 0,000599484 \\
\hline k141_74774 & Gamma-glutamyl hydrolase (EC 3.4.19.9) & 4,29 & 1,74 & 1,38 & 0,005247935 \\
\hline k141_481081 & Glycerol-3-phosphate transporter & 2,23 & 265,17 & 0,24 & $3,95873 E-19$ \\
\hline k141_224399 & Glycerol-3-phosphate transporter & 2,01 & 40,10 & 0,25 & $4,92828 \mathrm{E}-14$ \\
\hline k141_193668 & Glycerol-3-phosphate transporter & 2,32 & 18,62 & 0,33 & $2,22057 \mathrm{E}-11$ \\
\hline k141_389014 & Glycerol-3-phosphate transporter & $-0,31$ & 3154,59 & 0,08 & 0,000447475 \\
\hline k141_457268 & Glycerol-3-phosphate transporter & 4,49 & 2,02 & 1,23 & 0,000895182 \\
\hline k141_425508 & Glycerol-3-phosphate transporter & $-1,53$ & 10,83 & 0,42 & 0,000997363 \\
\hline k141_146839 & Hydroxymethylglutaryl-CoA lyase (EC 4.1.3.4) & 4,68 & 6,52 & 0,90 & $1,5418 \mathrm{E}-06$ \\
\hline k141_139861 & Hydroxymethylglutaryl-CoA lyase (EC 4.1.3.4) & $-1,13$ & 2051,92 & 0,22 & $2,22582 \mathrm{E}-06$ \\
\hline k141_502140 & Hydroxymethylglutaryl-CoA lyase (EC 4.1.3.4) & $-4,41$ & 1,93 & 1,14 & 0,000425189 \\
\hline k141_510582 & Hydroxymethylglutaryl-CoA lyase (EC 4.1.3.4) & 1,39 & 15,18 & 0,42 & 0,002557706 \\
\hline k141_230741 & Hydroxymethylglutaryl-CoA lyase (EC 4.1.3.4) & 2,11 & 2,96 & 0,72 & 0,009191594 \\
\hline k141_125817 & Inosine-uridine preferring nucleoside & $-0,60$ & 8875,48 & 0,09 & $1,2322 \mathrm{E}-10$ \\
\hline k141_34024 & Inosine-uridine preferring nucleoside & 4,51 & 7,55 & 0,73 & $6,58206 \mathrm{E}-09$ \\
\hline k141_67379 & Inosine-uridine preferring nucleoside & $-5,27$ & 3,48 & 1,07 & $5,37464 \mathrm{E}-06$ \\
\hline k141_237094 & Inosine-uridine preferring nucleoside & 2,52 & 9,44 & 0,55 & $2,58737 \mathrm{E}-05$ \\
\hline k141_357842 & Inosine-uridine preferring nucleoside & $-3,92$ & 2,78 & 0,89 & $5,04037 \mathrm{E}-05$ \\
\hline k141_543936 & Inosine-uridine preferring nucleoside & 4,29 & 2,46 & 0,97 & $5,42924 \mathrm{E}-05$ \\
\hline k141_1288 & Inosine-uridine preferring nucleoside & 4,01 & 2,92 & 1,00 & 0,000277133 \\
\hline k141_253294 & Inosine-uridine preferring nucleoside & 3,33 & 9,72 & 1,04 & 0,003892242 \\
\hline
\end{tabular}


Supplementary Table S5

\begin{tabular}{|c|c|c|c|c|c|}
\hline ORF & Rank3 & $\begin{array}{l}\text { Log2Fold } \\
\text { Change }\end{array}$ & base Mean & IfcSE & padj \\
\hline k141_76955 & Inosine-uridine preferring nucleoside & 2,81 & 5,23 & 0,97 & 0,009333198 \\
\hline k141_355623 & L-Proline/Glycine betaine transporter ProP & 3,92 & 9,01 & 0,79 & $3,90087 \mathrm{E}-06$ \\
\hline k141_65112 & L-Proline/Glycine betaine transporter ProP & 3,28 & 12,83 & 0,83 & 0,000325879 \\
\hline k141_215212 & L-Proline/Glycine betaine transporter ProP & 3,79 & 4,76 & 1,08 & 0,001444126 \\
\hline k141_559550 & L-Proline/Glycine betaine transporter ProP & $-4,11$ & 1,55 & 1,22 & 0,002426106 \\
\hline k141_498191 & L-Proline/Glycine betaine transporter ProP & $-1,99$ & 3,46 & 0,68 & 0,00891984 \\
\hline k141_416324 & photosystem I subunit II (PsaD) & 2,58 & 413,91 & 0,21 & $3,74569 \mathrm{E}-32$ \\
\hline k141_535769 & photosystem I subunit II (PsaD) & $-1,54$ & 443,63 & 0,13 & $4,10825 E-29$ \\
\hline k141_199899 & photosystem I subunit II (PsaD) & 7,44 & 74,50 & 0,66 & $1,26862 \mathrm{E}-27$ \\
\hline k141_247909 & photosystem I subunit II (PsaD) & $-2,51$ & 72,70 & 0,29 & $7,72722 \mathrm{E}-17$ \\
\hline k141_565346 & photosystem I subunit II (PsaD) & $-1,33$ & 170,46 & 0,17 & $5,50027 \mathrm{E}-14$ \\
\hline k141_235587 & photosystem I subunit II (PsaD) & 1,98 & 1351,95 & 0,27 & $3,54144 \mathrm{E}-12$ \\
\hline k141_73751 & photosystem I subunit II (PsaD) & 1,60 & 28,00 & 0,27 & $2,60764 \mathrm{E}-08$ \\
\hline k141_266241 & photosystem I subunit II (PsaD) & $-1,44$ & 58,85 & 0,24 & $3,80212 \mathrm{E}-08$ \\
\hline k141_566820 & photosystem I subunit II (PsaD) & 2,94 & 7,21 & 0,58 & 2,99159E-06 \\
\hline k141_445585 & photosystem I subunit II (PsaD) & $-0,57$ & 160,33 & 0,16 & 0,001142777 \\
\hline k141_104668 & photosystem I subunit II (PsaD) & 3,20 & 2,45 & 0,94 & 0,002100047 \\
\hline k141_207282 & photosystem I subunit II (PsaD) & 3,41 & 3,43 & 1,12 & 0,006501858 \\
\hline k141_98502 & $\begin{array}{l}\text { photosystem I subunit III precursor, } \\
\text { plastocyanin (cyt c553) docking protein (PsaF) }\end{array}$ & 2,38 & 403,48 & 0,22 & $6,02003 E-25$ \\
\hline k141_93528 & $\begin{array}{l}\text { photosystem I subunit III precursor, } \\
\text { plastocyanin (cyt c553) docking protein (PsaF) }\end{array}$ & 2,00 & 761,02 & 0,27 & $1,77381 \mathrm{E}-12$ \\
\hline k141_208417 & $\begin{array}{l}\text { photosystem I subunit III precursor, } \\
\text { plastocyanin (cyt c553) docking protein (PsaF) }\end{array}$ & $-0,72$ & 742,75 & 0,10 & $6,38572 \mathrm{E}-11$ \\
\hline k141_464550 & $\begin{array}{l}\text { photosystem I subunit III precursor, } \\
\text { plastocyanin (cyt c553) docking protein (PsaF) }\end{array}$ & $-1,54$ & 47,06 & 0,23 & $1,44424 \mathrm{E}-10$ \\
\hline k141_522821 & $\begin{array}{l}\text { photosystem I subunit III precursor, } \\
\text { plastocyanin (cyt c553) docking protein (PsaF) }\end{array}$ & $-2,65$ & 36,44 & 0,41 & $1,28445 \mathrm{E}-09$ \\
\hline k141_295117 & $\begin{array}{l}\text { photosystem I subunit III precursor, } \\
\text { plastocyanin (cyt c553) docking protein (PsaF) }\end{array}$ & 1,75 & 845,12 & 0,29 & $2,57856 \mathrm{E}-08$ \\
\hline k141_398369 & $\begin{array}{l}\text { photosystem I subunit III precursor, } \\
\text { plastocyanin (cyt c553) docking protein (PsaF) }\end{array}$ & $-1,40$ & 103,70 & 0,24 & $7,4738 \mathrm{E}-08$ \\
\hline k141_3413 & $\begin{array}{l}\text { photosystem I subunit III precursor, } \\
\text { plastocyanin (cyt c553) docking protein (PsaF) }\end{array}$ & $-0,66$ & 295,07 & 0,12 & $9,42142 \mathrm{E}-08$ \\
\hline k141_332077 & $\begin{array}{l}\text { photosystem I subunit III precursor, } \\
\text { plastocyanin (cyt c553) docking protein (PsaF) }\end{array}$ & $-1,56$ & 239,84 & 0,31 & $4,74773 E-06$ \\
\hline k141_138596 & $\begin{array}{l}\text { photosystem I subunit III precursor, } \\
\text { plastocyanin (cyt c553) docking protein (PsaF) }\end{array}$ & 2,53 & 5,46 & 0,60 & 0,000121784 \\
\hline k141_577144 & $\begin{array}{l}\text { photosystem I subunit III precursor, } \\
\text { plastocyanin (cyt c553) docking protein (PsaF) }\end{array}$ & $-1,77$ & 8,68 & 0,49 & 0,000912868 \\
\hline k141_138301 & $\begin{array}{l}\text { photosystem I subunit III precursor, } \\
\text { plastocyanin (cyt c553) docking protein (PsaF) }\end{array}$ & 3,11 & 2,34 & 0,86 & 0,001030409 \\
\hline k141_87224 & $\begin{array}{l}\text { photosystem I subunit III precursor, } \\
\text { plastocyanin (cyt c553) docking protein (PsaF) }\end{array}$ & 2,44 & 3,24 & 0,78 & 0,005159722 \\
\hline k141_13050 & photosystem I subunit PsaO & 2,58 & 199,31 & 0,21 & $4,83136 \mathrm{E}-32$ \\
\hline k141_432749 & photosystem I subunit PsaO & $-1,12$ & 441,89 & 0,12 & $2,82635 \mathrm{E}-20$ \\
\hline k141_578984 & photosystem I subunit PsaO & $-1,27$ & 434,08 & 0,15 & $8,98056 \mathrm{E}-16$ \\
\hline k141_279621 & photosystem I subunit PsaO & 2,07 & 183,93 & 0,31 & $5,08598 \mathrm{E}-10$ \\
\hline k141_364340 & photosystem I subunit PsaO & $-0,87$ & 256,33 & 0,13 & $1,02666 \mathrm{E}-09$ \\
\hline k141_177301 & photosystem I subunit PsaO & 1,99 & 456,74 & 0,31 & $2,19456 \mathrm{E}-09$ \\
\hline k141_266794 & photosystem I subunit PsaO & $-1,42$ & 64,67 & 0,25 & $1,38501 \mathrm{E}-07$ \\
\hline k141_163809 & photosystem I subunit PsaO & $-1,56$ & 297,28 & 0,39 & 0,000274167 \\
\hline
\end{tabular}




\section{Supplementary Table S5}

\begin{tabular}{ll}
\multicolumn{1}{c}{ ORF } & Rank3 \\
k141_1476 & photosystem I subunit X (PsaK, PsaK1) \\
k141_518292 & photosystem I subunit X (PsaK, PsaK1) \\
k141_312007 & photosystem I subunit X (PsaK, PsaK1) \\
k141_537570 & photosystem I subunit X (PsaK, PsaK1) \\
k141_339993 & photosystem I subunit X (PsaK, PsaK1) \\
k141_455562 & photosystem I subunit XI (PsaL) \\
k141_190608 & photosystem I subunit XI (PsaL) \\
k141_334187 & photosystem I subunit XI (PsaL)
\end{tabular}

k141_228666

k141_130801

k141_223209

k141_489633

k141_268980

k141_345504

k141_346335

k141_507137

k141_195871

k141_422781

k141_326733

k141_368506

k141_29951

k141_345096

k141_539900

k141_557213

k141_324219

k141_60116

k141_564911

k141_23099

k141_44560

k141_503320

k141_520673

k141_297088

k141_194023

k141_176594

k141_207737

k141_70397

k141_437270

k141_485748

k141_384269

k141_89459

k141_361938

k141_335555

k141_412498

k141_434083

k141_28060

k141_22624

k141_177671

k141_323426

k141_461167

k141_317349

\begin{tabular}{|c|c|c|c|}
\hline \multicolumn{4}{|l|}{ Log2Fold } \\
\hline Change & base Mean & IfcSE & padj \\
\hline 2,70 & 240,69 & 0,26 & $6,20259 E-23$ \\
\hline$-1,44$ & 47,35 & 0,22 & $1,62293 \mathrm{E}-09$ \\
\hline 1,85 & 515,72 & 0,30 & $5,85316 \mathrm{E}-09$ \\
\hline 2,61 & 3,81 & 0,65 & 0,000216023 \\
\hline 3,44 & 2,04 & 0,96 & 0,001236292 \\
\hline$-8,58$ & 48,31 & 0,69 & $2,27354 \mathrm{E}-33$ \\
\hline 2,51 & 488,98 & 0,27 & $2,95535 \mathrm{E}-19$ \\
\hline 2,04 & 1026,47 & 0,28 & $1,09354 \mathrm{E}-11$ \\
\hline$-1,43$ & 57,81 & 0,24 & $2,35541 \mathrm{E}-08$ \\
\hline$-2,68$ & 7,10 & 0,57 & 1,6285E-05 \\
\hline 3,35 & 1,95 & 0,93 & 0,001124451 \\
\hline$-1,15$ & 70,39 & 0,32 & 0,001262853 \\
\hline 2,96 & 3,67 & 0,86 & 0,001975307 \\
\hline 2,07 & 49,94 & 0,28 & $4,9786 \mathrm{E}-12$ \\
\hline 1,91 & 149,55 & 0,27 & $1,56695 \mathrm{E}-11$ \\
\hline 3,84 & 17,85 & 0,86 & $4,37599 \mathrm{E}-05$ \\
\hline 4,05 & 3,03 & 0,94 & $8,19761 \mathrm{E}-05$ \\
\hline 4,56 & 2,10 & 1,10 & 0,000153749 \\
\hline 0,98 & 68,92 & 0,25 & 0,000270002 \\
\hline$-0,63$ & 860,68 & 0,16 & 0,000457751 \\
\hline$-1,61$ & 8,90 & 0,47 & 0,001842017 \\
\hline 2,10 & 3,56 & 0,67 & 0,00472691 \\
\hline$-5,11$ & 40,18 & 0,33 & $2,43983 E-51$ \\
\hline$-1,51$ & 76,40 & 0,17 & $4,08634 \mathrm{E}-18$ \\
\hline 2,18 & 188,96 & 0,25 & $1,28753 \mathrm{E}-16$ \\
\hline$-1,35$ & 357,90 & 0,18 & $4,44854 \mathrm{E}-13$ \\
\hline$-5,32$ & 3,59 & 0,84 & $2,43494 \mathrm{E}-09$ \\
\hline 4,02 & 4,10 & 0,85 & $1,34675 \mathrm{E}-05$ \\
\hline$-0,41$ & 1752,70 & 0,11 & 0,001241491 \\
\hline$-1,79$ & 60,92 & 0,52 & 0,001907702 \\
\hline$-3,14$ & 2,20 & 1,08 & 0,009658933 \\
\hline 2,93 & 975,71 & 0,24 & 1,4892E-31 \\
\hline$-1,21$ & 454,72 & 0,14 & $4,66468 \mathrm{E}-17$ \\
\hline$-1,90$ & 99,95 & 0,26 & $7,00361 \mathrm{E}-12$ \\
\hline 2,07 & 4,28 & 0,67 & 0,005674152 \\
\hline 2,84 & 5,46 & 0,94 & 0,006513891 \\
\hline$-0,61$ & 7465,76 & 0,09 & $3,82026 \mathrm{E}-10$ \\
\hline 1,12 & 50,58 & 0,23 & $6,39876 \mathrm{E}-06$ \\
\hline$-2,15$ & 3,65 & 0,60 & 0,001212572 \\
\hline 1,36 & 12,83 & 0,40 & 0,00231469 \\
\hline$-2,35$ & 2,40 & 0,75 & 0,004673889 \\
\hline 3,84 & 1820,06 & 0,23 & $6,06385 \mathrm{E}-62$ \\
\hline$-1,96$ & 375,58 & 0,13 & $1,31066 \mathrm{E}-46$ \\
\hline$-1,97$ & 295,25 & 0,14 & $3,78121 \mathrm{E}-43$ \\
\hline 3,27 & 755,07 & 0,23 & $9,71614 \mathrm{E}-43$ \\
\hline 3,18 & 1592,28 & 0,25 & $9,94082 E-34$ \\
\hline 2,93 & 1905,83 & 0,24 & $5,45526 \mathrm{E}-33$ \\
\hline$-1,71$ & 168,68 & 0,15 & $5,82237 \mathrm{E}-29$ \\
\hline$-1,19$ & 165,17 & 0,11 & $1,99631 \mathrm{E}-23$ \\
\hline$-6,94$ & 11,07 & 0,68 & $7,29111 \mathrm{E}-23$ \\
\hline
\end{tabular}


Supplementary Table S5

\begin{tabular}{|c|c|c|c|c|c|}
\hline ORF & Rank3 & $\begin{array}{c}\text { Log2Fold } \\
\text { Change }\end{array}$ & base Mean & IfcSE & padj \\
\hline k141_35616 & Ribulose bisphosphate carboxylase small chain & 2,59 & 369,15 & 0,26 & $5,17756 \mathrm{E}-22$ \\
\hline k141_117874 & Ribulose bisphosphate carboxylase small chain & 2,24 & 121,05 & 0,22 & $7,8494 \mathrm{E}-22$ \\
\hline k141_276815 & Ribulose bisphosphate carboxylase small chain & 2,49 & 608,45 & 0,27 & $1,18614 \mathrm{E}-18$ \\
\hline k141_27483 & Ribulose bisphosphate carboxylase small chain & 2,51 & 12457,43 & 0,28 & $3,5801 \mathrm{E}-18$ \\
\hline k141_104258 & Ribulose bisphosphate carboxylase small chain & $-6,32$ & 19,29 & 0,71 & $1,46018 \mathrm{E}-17$ \\
\hline k141_268674 & Ribulose bisphosphate carboxylase small chain & 2,49 & 1116,96 & 0,28 & $3,22833 \mathrm{E}-17$ \\
\hline k141_27789 & Ribulose bisphosphate carboxylase small chain & 1,79 & 4879,06 & 0,21 & $1,79846 \mathrm{E}-16$ \\
\hline k141_50257 & Ribulose bisphosphate carboxylase small chain & 3,81 & 11,57 & 0,46 & $6,52962 \mathrm{E}-15$ \\
\hline k141_436841 & Ribulose bisphosphate carboxylase small chain & 2,66 & 29,06 & 0,35 & $3,77875 \mathrm{E}-13$ \\
\hline k141_162540 & Ribulose bisphosphate carboxylase small chain & $-1,47$ & 962,20 & 0,20 & $7,84327 \mathrm{E}-12$ \\
\hline k141_125305 & Ribulose bisphosphate carboxylase small chain & $-1,23$ & 326,57 & 0,17 & $8,52089 \mathrm{E}-12$ \\
\hline k141_75390 & Ribulose bisphosphate carboxylase small chain & 1,76 & 261,90 & 0,25 & $7,07346 \mathrm{E}-11$ \\
\hline k141_498912 & Ribulose bisphosphate carboxylase small chain & 4,44 & 10,52 & 0,65 & $1,26009 \mathrm{E}-10$ \\
\hline k141_394558 & Ribulose bisphosphate carboxylase small chain & 1,72 & 440,22 & 0,25 & $1,6039 \mathrm{E}-10$ \\
\hline k141_267978 & Ribulose bisphosphate carboxylase small chain & 2,04 & 1634,96 & 0,30 & $1,7359 \mathrm{E}-10$ \\
\hline k141_208364 & Ribulose bisphosphate carboxylase small chain & $-0,79$ & 1538,83 & 0,12 & $2,11571 \mathrm{E}-1 \mathrm{C}$ \\
\hline k141_260136 & Ribulose bisphosphate carboxylase small chain & 3,87 & 8,54 & 0,58 & $2,62989 \mathrm{E}-10$ \\
\hline k141_438106 & Ribulose bisphosphate carboxylase small chain & 2,94 & 11,62 & 0,44 & $4,18627 \mathrm{E}-10$ \\
\hline k141_119881 & Ribulose bisphosphate carboxylase small chain & $-1,07$ & 739,46 & 0,17 & 1,0905E-09 \\
\hline k141_357404 & Ribulose bisphosphate carboxylase small chain & $-4,48$ & 5,69 & 0,70 & $1,56366 \mathrm{E}-0 \Omega$ \\
\hline k141_17347 & Ribulose bisphosphate carboxylase small chain & 2,78 & 11,04 & 0,44 & $3,31128 \mathrm{E}-0 \mathrm{~s}$ \\
\hline k141_484488 & Ribulose bisphosphate carboxylase small chain & 2,08 & 39,77 & 0,33 & $3,37734 \mathrm{E}-0 \Omega$ \\
\hline k141_532535 & Ribulose bisphosphate carboxylase small chain & $-3,81$ & 18,23 & 0,61 & $4,70946 \mathrm{E}-0 \Omega$ \\
\hline k141_97867 & Ribulose bisphosphate carboxylase small chain & $-1,33$ & 654,03 & 0,21 & $6,07163 \mathrm{E}-0 \Omega$ \\
\hline k141_398759 & Ribulose bisphosphate carboxylase small chain & 3,24 & 12,53 & 0,53 & $9,57611 \mathrm{E}-0 \Omega$ \\
\hline k141_326291 & Ribulose bisphosphate carboxylase small chain & 1,62 & 325,24 & 0,28 & $5,40512 \mathrm{E}-08$ \\
\hline k141_542033 & Ribulose bisphosphate carboxylase small chain & 2,65 & 21,62 & 0,46 & $6,95043 \mathrm{E}-08$ \\
\hline k141_520986 & Ribulose bisphosphate carboxylase small chain & $-3,92$ & 3,90 & 0,74 & $9,10371 \mathrm{E}-07$ \\
\hline k141_564046 & Ribulose bisphosphate carboxylase small chain & 2,66 & 8,09 & 0,51 & $1,13718 \mathrm{E}-06$ \\
\hline k141_548356 & Ribulose bisphosphate carboxylase small chain & $-1,44$ & 28,96 & 0,28 & $1,81944 \mathrm{E}-06$ \\
\hline k141_500438 & Ribulose bisphosphate carboxylase small chain & $-0,76$ & 1492,40 & 0,16 & $1,79749 \mathrm{E}-05$ \\
\hline k141_458236 & Ribulose bisphosphate carboxylase small chain & 4,26 & 2,51 & 0,92 & $2,08526 \mathrm{E}-05$ \\
\hline k141_123668 & Ribulose bisphosphate carboxylase small chain & 1,58 & 28,45 & 0,34 & $2,19669 \mathrm{E}-05$ \\
\hline k141_45870 & Ribulose bisphosphate carboxylase small chain & 2,46 & 12,90 & 0,55 & $4,54645 \mathrm{E}-05$ \\
\hline k141_187455 & Ribulose bisphosphate carboxylase small chain & $-0,92$ & 44,82 & 0,21 & $6,42205 \mathrm{E}-05$ \\
\hline k141_44305 & Ribulose bisphosphate carboxylase small chain & $-0,64$ & 5918,56 & 0,15 & $6,70447 \mathrm{E}-05$ \\
\hline k141_508172 & Ribulose bisphosphate carboxylase small chain & $-1,40$ & 17,19 & 0,35 & 0,000308423 \\
\hline k141_420712 & Ribulose bisphosphate carboxylase small chain & 0,49 & 507,38 & 0,13 & 0,000351997 \\
\hline k141_288531 & Ribulose bisphosphate carboxylase small chain & 7,16 & 12,76 & 1,95 & 0,000867456 \\
\hline k141_180320 & Ribulose bisphosphate carboxylase small chain & 0,45 & 562,35 & 0,12 & 0,00106172 \\
\hline k141_56135 & Ribulose bisphosphate carboxylase small chain & 1,34 & 11,35 & 0,37 & 0,001121168 \\
\hline k141_53869 & Ribulose bisphosphate carboxylase small chain & 0,85 & 4119,67 & 0,26 & 0,002775729 \\
\hline k141_283875 & Ribulose bisphosphate carboxylase small chain & 0,39 & 1820,75 & 0,12 & 0,002785151 \\
\hline k141_377448 & Ribulose bisphosphate carboxylase small chain & $-1,24$ & 404,83 & 0,37 & 0,002802405 \\
\hline k141_186696 & Ribulose bisphosphate carboxylase small chain & 3,63 & 2,30 & 1,12 & 0,003505223 \\
\hline k141_373016 & Ribulose bisphosphate carboxylase small chain & 0,39 & 1100,02 & 0,12 & 0,00355818 \\
\hline k141_339217 & Ribulose bisphosphate carboxylase small chain & $-1,95$ & 15,19 & 0,60 & 0,003593552 \\
\hline k141_291576 & Ribulose bisphosphate carboxylase small chain & 0,38 & 1946,95 & 0,12 & 0,00399674 \\
\hline k141_418272 & Ribulose bisphosphate carboxylase small chain & 0,33 & 8122,41 & 0,10 & $0,00407726 s$ \\
\hline k141_501798 & Ribulose bisphosphate carboxylase small chain & 0,32 & 3247,47 & 0,11 & 0,006033711 \\
\hline
\end{tabular}


Supplementary Table S5

\begin{tabular}{|c|c|c|c|c|c|}
\hline ORF & Rank3 & $\begin{array}{c}\text { Log2Fold } \\
\text { Change }\end{array}$ & base Mean & IfcSE & padj \\
\hline k141_193554 & Ribulose bisphosphate carboxylase small chain & 0,61 & 421,72 & 0,20 & 0,006589074 \\
\hline k141_399059 & S-formylglutathione hydrolase (EC 3.1.2.12) & $-6,50$ & 8,19 & 0,71 & $1,82928 \mathrm{E}-18$ \\
\hline k141_114710 & S-formylglutathione hydrolase (EC 3.1.2.12) & 2,28 & 21,22 & 0,41 & $2,27241 \mathrm{E}-07$ \\
\hline k141_399265 & S-formylglutathione hydrolase (EC 3.1.2.12) & $-5,31$ & 3,57 & 0,97 & $3,20614 \mathrm{E}-07$ \\
\hline k141_144658 & S-formylglutathione hydrolase (EC 3.1.2.12) & $-0,46$ & 1151,64 & 0,12 & 0,000295845 \\
\hline k141_549493 & S-formylglutathione hydrolase (EC 3.1.2.12) & 2,58 & 5,22 & 0,67 & 0,000507979 \\
\hline k141_541479 & S-formylglutathione hydrolase (EC 3.1.2.12) & 3,97 & 2,86 & 1,20 & 0,002839428 \\
\hline k141_422938 & Sucrose-6-phosphate hydrolase (EC 3.2.1.B3) & $-5,19$ & 23,69 & 0,56 & $1,42418 \mathrm{E}-18$ \\
\hline k141_390600 & Sucrose-6-phosphate hydrolase (EC 3.2.1.B3) & 3,50 & 22,42 & 0,44 & $3,03372 \mathrm{E}-14$ \\
\hline k141_17690 & Sucrose-6-phosphate hydrolase (EC 3.2.1.B3) & 3,09 & 26,00 & 0,41 & $4,92529 \mathrm{E}-13$ \\
\hline k141_330797 & Sucrose-6-phosphate hydrolase (EC 3.2.1.B3) & $-1,31$ & 358,84 & 0,19 & $4,44635 \mathrm{E}-11$ \\
\hline k141_83755 & Sucrose-6-phosphate hydrolase (EC 3.2.1.B3) & 4,79 & 5,84 & 0,87 & $2,65083 \mathrm{E}-07$ \\
\hline k141_556054 & Sucrose-6-phosphate hydrolase (EC 3.2.1.B3) & 2,65 & 9,73 & 0,53 & $3,10383 \mathrm{E}-06$ \\
\hline k141_121216 & Sucrose-6-phosphate hydrolase (EC 3.2.1.B3) & 2,88 & 4,97 & 0,68 & 0,000104656 \\
\hline k141_128619 & Sucrose-6-phosphate hydrolase (EC 3.2.1.B3) & 4,59 & 3,05 & 1,12 & 0,000171844 \\
\hline k141_525775 & Uridine diphosphate glucose pyrophosphatase & 1,58 & 16,23 & 0,42 & 0,0005962 \\
\hline
\end{tabular}

